\title{
L-DOPA-Induced Dyskinesia in a Genetic Drosophila Model of Parkinson's Disease
}

\author{
Joshua A. Blosser, Eric Podolsky and Daewoo Lee* \\ Neuroscience Program, Department of Biological Sciences, Ohio University, Athens, OH 45701, USA
}

\begin{abstract}
Motor symptoms in Parkinson's disease (PD) are directly related to the reduction of a neurotransmitter dopamine. Therefore, its precursor LDOPA became the gold standard for PD treatment. However, chronic use of L-DOPA causes uncontrollable, involuntary movements, called LDOPA-induced dyskinesia (LID) in the majority of PD patients. LID is complicated and very difficult to manage. Current rodent and non-human primate models have been developed to study LID mainly using neurotoxins. Therefore, it is necessary to develop a LID animal model with defects in genetic factors causing PD in order to study the relation between LID and PD genes such as $\alpha$-synuclein. In this study, we first showed that a low concentration of L-DOPA $(100 \mu \mathrm{M})$ rescues locomotion defects (i.e., speed, angular velocity, pause time) in Drosophila larvae expressing human mutant $\alpha$-synuclein (A53T). This A53T larval model of PD was used to further examine dyskinetic behaviors. High concentrations of L-DOPA (5 or $10 \mathrm{mM}$ ) causes hyperactivity such as body bending behavior (BBB) in A53T larva, which resembles axial dyskinesia in rodents. Using ImageJ plugins and other third party software, dyskinetic BBB has been accurately and efficiently quantified. Further, we showed that a dopamine agonist pramipexole (PRX) partially rescues BBB caused by high L-DOPA. Our Drosophila genetic LID model will provide an important experimental platform to examine molecular and cellular mechanisms underlying LID, to study the role of PD causing genes in the development of LID, and to identify potential targets to slow/reverse LID pathology.
\end{abstract}

Key words: Alpha-Synuclein, Parkinson's disease, L-DOPA, Dyskinesia, Drosophila melanogaster

\section{INTRODUCTION}

There is no cure for Parkinson's disease (PD) in spite of intensive research for last decades. Nonetheless, several drugs have been used to ameliorate PD symptoms. L-DOPA became a gold standard drug to treat PD symptoms. However, chronic use of LDOPA for 5 10 years causes uncontrollable movements, called LDOPA-induced dyskinesia (LID) in the majority of PD patients [1, 2]. LID is very difficult to manage and complicated to understand. Therefore, animal models have been developed to seek the mechanisms involved in LID and identify new drug targets $[3,4]$. Cur-

Submitted July 11,2020, Revised August 27, 2020,

Accepted August 31,2020

* To whom correspondence should be addressed. TEL: 1-740-597-1926, FAX: 1-740-593-0300 e-mail: Leed1@ohio.edu rent rodent and non-human primate models have been developed mainly using MPTP and 6-OHDA neurotoxins. In addition to neurotoxins, genetic factors (e.g., a-synuclein, parkin, and LRRK2) are involved in PD $[5,6]$. Since the discovery of the first PD gene a-synuclein ( $a$-Syn), the genetic PD models have provided key insights into disease mechanisms. Therefore, it is necessary to develop a LID animal model with defects in these PD genes to study the relation between LID and these genes such as a-Syn. However, genetically tractable mouse models are known to poorly recapitulate PD symptoms (e.g., no degeneration of DA neurons) [7, 8], and thus it is urgent and necessary to develop a new genetic PD model showing LID.

The fruit fly Drosophila melanogaster has been a highly useful genetic model of human neural diseases. In addition to the shortgeneration time and dramatically reduced costs of maintaining large populations, the ability to combine behavioral and biochemical approaches with sophisticated molecular genetics has made 
Drosophila a 'model organism of choice' for identification of genes involved in higher brain function and disease pathogenesis. Perhaps surprisingly to some, gene sequences and function are highly conserved between flies, rodents and humans $[9,10]$. About two thirds of human disease gene homologues are found in the Drosophila genome. Furthermore, a humanized fly disease model can be easily created using a transgenic fly expressing a mutant human disease gene (e.g. mutant human $a-S y n)$ [11-13]. Drosophila $\alpha$-Syn flies replicate all major PD symptoms: selective loss of dopaminergic (DA) neurons, intracellular aggregates called Lewy Bodies, and locomotion defects [11-15]. Therefore, mutant $a$-Syn Drosophila can be an excellent genetic PD model to study LID.

In order to develop an LID model, it is necessary to confirm that PD symptoms can be rescued by L-DOPA. Next step is to study whether prolonged or high L-DOPA administration causes dyskinetic behavior. In this study, we showed that a low concentration of L-DOPA $(100 \mu \mathrm{M})$ rescues locomotion defects in Drosophila larvae expressing human mutant $\alpha$-Syn (A53T). Therefore, this fly larval model of PD was used to further examine dyskinetic behaviors. High concentrations ( 5 or $10 \mathrm{mM}$ ) of chronic L-DOPA administration showed hyperactivity in A53T larva such as body bending behavior (BBB), which resembles axial dyskinesia in rodents $[16,17]$. We also developed a method to accurately and efficiently quantify LID behavior via the use of ImageJ plugins and other third party software. Further, dyskinetic BBB by high LDOPA was rescued by a dopamine agonist pramipexole (PRX). Our LID model developed in a Drosophila genetic model of PD can be a useful experimental platform to study pathophysiological mechanisms underlying LID and also provide a new avenue for developing potential treatments of LID.

\section{MATERIALS AND METHODS}

\section{Fly strains}

Flies were grown in standard cornmeal/agar media with $0.4 \%$ propionic acid on a 12 -hour light/dark cycle at $25^{\circ} \mathrm{C}$. Fly strains used were: wild type w1118 (from Bloomington Stock Center), TH-Gal4 (a kind gift from Dr S. Birman at ESPCI, France) [18], UAS-a-Syn (A53T) (A53T, a gift from Dr. L. Pallanck, University of Washington, USA) [19]. In this study, we used a fly line carrying TH-Gal4 and UAS- $\alpha-S y n$ (A53T) on chromosome 3, designated as A53T. Flies were allowed to lay eggs onto an agar plate for an hour. Each plate had roughly 50 eggs on its surface. The plate was then incubated at $25^{\circ} \mathrm{C}$ for locomotion assay.

\section{Larval locomotion assay}

Larvae were collected at 90 96 hours after egg laying for the locomotion assay as previously described [15]. An assay stage was prepared by boiling 2.5\% agar in ddH2O, adding 5 drops of India ink for contrast, and pouring the mixture into a petri dish to solidify. Third instar larvae were loosened from the food using dd $\mathrm{H} 2 \mathrm{O}$ and placed individually on the stage to be tested. First, the larvae were allowed to move freely for one minute to let them acclimate to the stage. Next, the larva was recorded using a Moticam 3 digital camera recording at approximately 10 frames/sec with the Motic Images Plus 2.0 software. The recording time was 30 seconds, with recording being stopped if the larva left the field of view (FOV). Several drops of ddH2O were periodically added to the stage to keep it from drying.

L-DOPA was added to the melted food in the egg laying plates before solidifying and larvae were incubated in this food for the entire 90-96 hour period or 48 hours during the third instar stage before testing. L-DOPA was administered in food with ascorbic acid $(25 \mathrm{mg} / 100 \mathrm{ml})$ to prevent oxidation [20]. Stock solutions were prepared monthly and kept at $4^{\circ} \mathrm{C}$ to ensure freshness. All LDOPA controls contained matching amounts of ascorbic acid.

\section{Analysis of locomotion parameters}

The program ImageJ (http://rsb.info.nih.gov/ij/) was used to analyze the video files. The videos were first imported to produce a stack of images for each frame. The color threshold was then adjusted so that the larva was a distinct object in contrast with the background. In order to determine the locomotion speed of the larvae, the MTrack2 plug-in (http://valelab.ucsf.edu/ nico/ IJplugins/MTrack2.html) was used to determine the path length. The length was then converted to $\mathrm{mm} / \mathrm{min}$ by using the number of seconds in the video file as well as a conversion factor between pixels and millimeters (12.2 pixels $/ \mathrm{mm}$ ), which had been determined to be given the height of the camera as previously adopted [15].

To find the angular speed and pause time of the larvae, software was developed (with the assistance of J. Bury, B.S., Computer Science, University of Toledo, USA) to analyze the raw movement data as exported from MTrack2. The raw data consisted of a set of ordered pairs corresponding to all the points to which the larva moved. To analyze the raw data, the software divided the number of frames in the video by the number of seconds in the video. The resulting number corresponded to the number of frames in one second of video, and the software then sampled the position of the larva at each second. This was done to reduce noise in the video to provide more accurate results. The camera used in this study recorded at 16 frames per second. Lines were then made connecting each of the selected points (Fig. 1A). To determine the angular speed, the angle was determined at the intersection of each pair of 
A

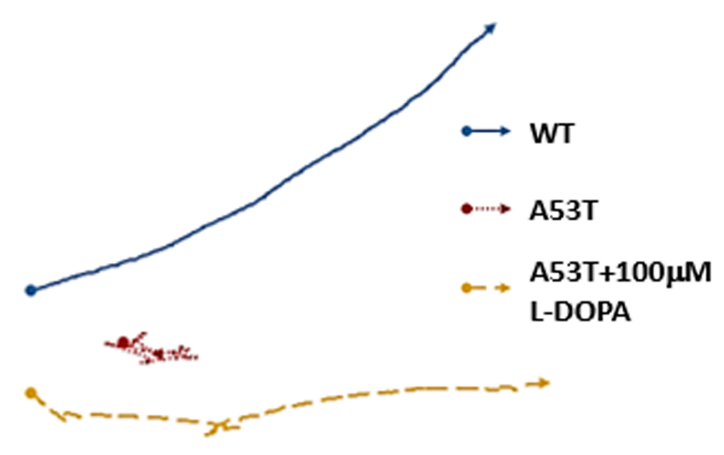

C

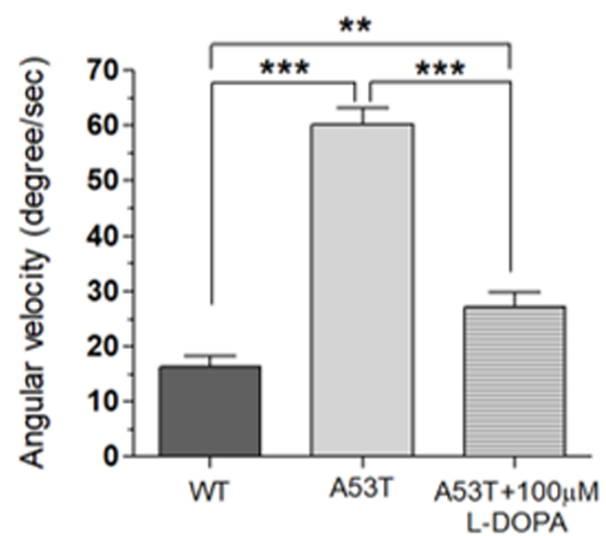

B

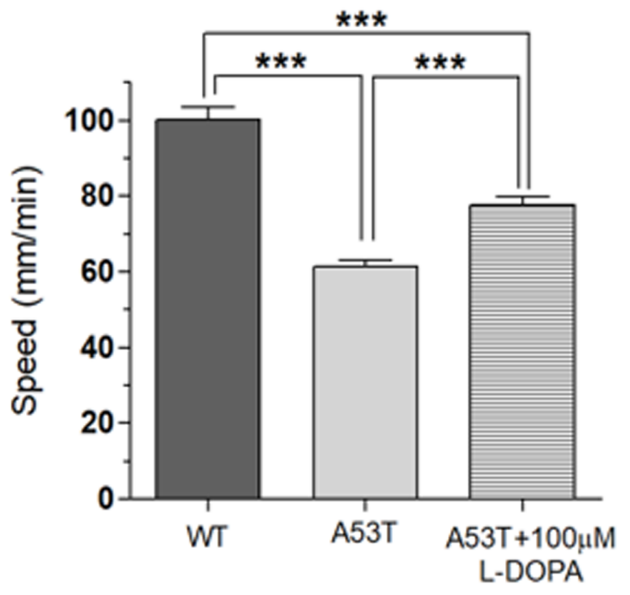

D

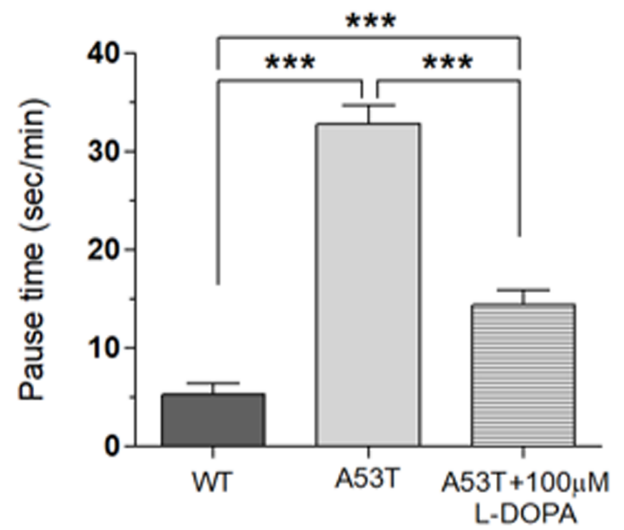

Fig. 1. L-DOPA rescues locomotion defects in $\alpha$-Syn (A53T) Drosophila model. Fly larvae expressing $\alpha-S y n$ (A53T) showed locomotion defects quantified with three locomotion parameters: speed, angular velocity, and pause time. (A) Crawling paths were visualized by selecting the 30 second paths that most closely represents the locomotion parameters of each group. Arrows were placed at the end of each pathway to show the direction of motion. When $100 \mu \mathrm{M}$ L-DOPA were applied to A53T larvae for 92 hours, all three locomotive parameters including speed (B), angular velocity (C), and pause time (D) were rescued. Note that rescue was not complete, as there was still a significant difference between flies treated with L-DOPA and wild type flies. Student t-test, ${ }^{* *} \mathrm{p}<0.01,{ }^{* * *} \mathrm{p}<0.001$. For each group, 30 larvae from 4 separate experiments were examined.

lines. To do this, the distance formula (Equation 1) was used to determine the distance of each of the two lines, as well as the distance of a third line that would produce a triangle between the two lines.

Equation 1: $\quad d=\sqrt{\left(x_{2}-x_{1}\right)^{2}+\left(y_{2}-y_{1}\right)^{2}}$

Given this triangle, the angle of interest $(\gamma)$ was determined using the law of cosines (Equation 2).

Equation 2: $\quad c^{2}=a^{2}+b^{2}-2 a b * \cos (\gamma)$

This angle was then subtracted from $180^{\circ}$ to determine how many degrees the larva had deviated from a straight line in that given second. This analysis was done for each second, and the average in degree $\left(^{\circ}\right)$ /sec was subsequently determined by divid- ing the sum of all of the angles by the number of seconds. The pause time was determined using the same software. For each line indicating a second of movement, the distance was converted to $\mathrm{mm} / \mathrm{min}$ using the technique described above. A threshold value would determine whether the larva was considered to be "paused" or "in motion" as even a stopped larva registers some movement due to head shaking, rolling, etc. A value of $45 \mathrm{~mm} / \mathrm{min}$ was found to correlate well with the pause time seen by a human observer for several videos. The number of seconds in which the larva moved at a speed less than the threshold value $(45 \mathrm{~mm} / \mathrm{min})$ was found, and this was then converted to the number of seconds paused per minute.

\section{Quantifying body bending behavior}

Our method involves keeping a larva in a small well $(\phi=2 \mathrm{~mm})$ 
Joshua A. Blosser, et al.

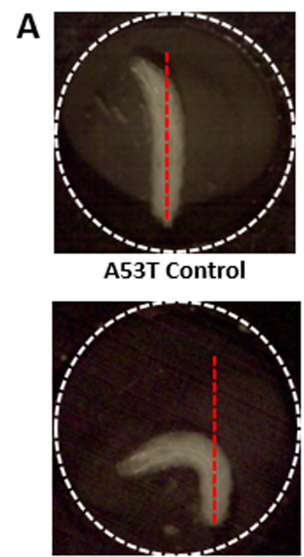

B

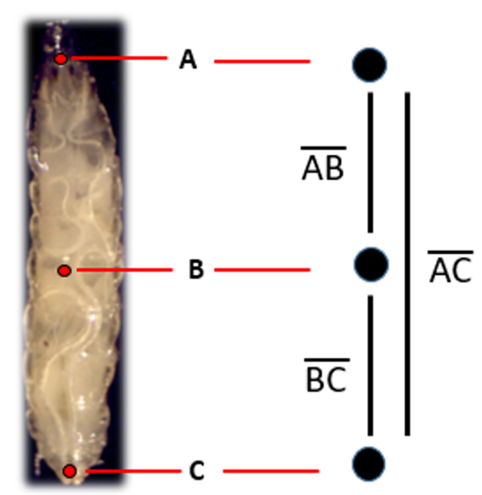

C

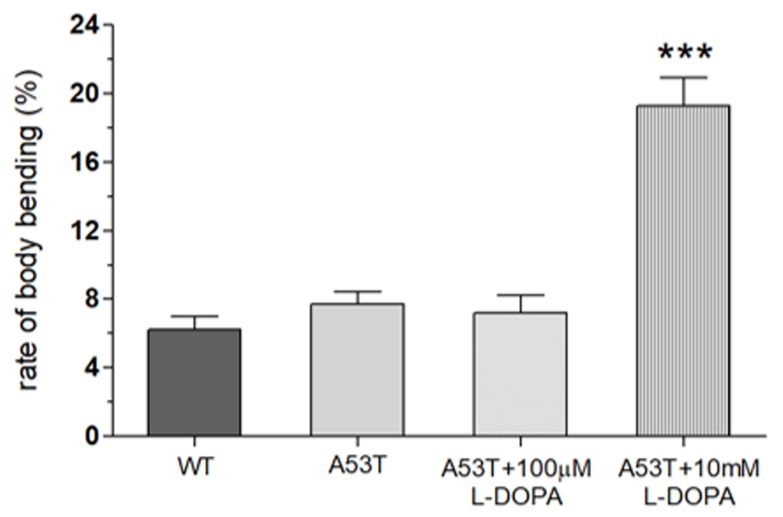

A53T + 10mM L-DOPA

Fig. 2. L-DOPA-induced dyskinesia in A53T Drosophila larvae. (A) Example snapshots of body bending behavior (BBB) in A53T Drosophila larvae treated with high concentration of L-DOPA (A53T+10 mM L-DOPA) or without L-DOPA (A53T Control). A larva was kept in a well (2 mm diameter) to limit free-crawling and thus enable us to analyze body bending behavior (BBB). A well is delineated with a dotted circle. (B) Three points were selected at the head, midpoint, and tail of the larva on every frame in the sub-stack, creating a three point skeleton which can be used to quantify BBB (see Materials and Methods). (C) Larvae exposed to a high concentration (10 mM) of L-DOPA display a higher body bending percentage, but A53T larvae with low concentration of L-DOPA $(\mathrm{A} 53 \mathrm{~T}+100 \mu \mathrm{M})$ or without L-DOPA (A53T Control) did not show changes in BBB, demonstrating dyskinetic behaviors in a genetic PD model exposed to high concentration of L-DOPA. Student t-test, ${ }^{* * *} \mathrm{p}<0.001$. For each group, $27 \sim 32$ larvae from 8 separate experiments were examined.

that is slightly larger than the length of the larva (Fig. 2A), inhibiting the larvae from any lateral crawling motion, but only allows movement along the body axis of the larva and around the vertical axis of the well. To analyze body bending behavior (BBB), $30 \mathrm{sec}-$ ond video was taken of a larva in the well and then imported into ImageJ to create a stack. Sub-stacks were created by selecting every 32nd frame. As the MoticCam shoots 16 frames/second, this created a stack with one frame for every two seconds of video. The subsequent sub-stacks were then analyzed in ImageJ via the ManualTracking plugin. Three points, one on the head, midpoint, and tail, were tracked by manually selecting them on every frame in the sub-stack, reducing the body of the larva to a three-point skeleton (Fig. 2B). The distance formula (Equation 1) was used to compare the entire length of the larva to the distance from the head to the tail. The entire length of the larva was measured as the length of $\overline{A B}+\overline{B C}$, while the distance from head to tail was measured as the length of $\overline{A C}$. The rate of body bending was then calculated as a ratio of the length of $\overline{A B}+\overline{B C}$ to the length of $\overline{A C}$, via Equation 3.

Equation 3: $\quad$ rate of body bending $=1-\overline{A C} /(\overline{A B}+\overline{B C})$

As the body of the larva bent and the head was brought down towards the tail, $\overline{A C}$ decreased while $\overline{A B}+\overline{B C}$ stayed the same. As this ratio is a measure of overall straightness, the fraction was subtracted from one to get a measure of the decrease in straightness, or body bending behavior (BBB).

\section{Statistical analysis}

Data are shown as mean \pm SEM. Statistical analysis was performed using Graph-Pad Prism software. Means were compared using an unpaired two-tailed Student's t-test. $p$ values $\leq 0.05$ were considered significant.

\section{RESULTS}

\section{L-DOPA rescues $\alpha$-Syn-mediated locomotion defects}

Our previous study showed that the fruit fly Drosophila larva expressing human $\alpha$-Syn (A53T) crawls significantly slower compared to wild type fly larva [15]. A53T fly larva (TH-Gal4, UASa-SynA53T) also shows reduced number of dopaminergic (DA) neurons in the brain [15]. In addition to the speed, we also analyzed two additional motor behaviors: angular velocity and pause time. Since slow movement (bradykinesia) is only one of the motor symptoms of PD, and other measures of locomotion such as angular velocity and pause time have been measured. Locomotion speed represents bradykinesia, which is one of the major motor symptoms of PD, but angular velocity and pause time are used to see other behaviors related to PD symptoms such as walking difficulties and gait freezing. These three parameters are different ways to examine various PD-like symptoms.

Using UAS x Gal4 binary system [21], a mutant human $\alpha-S y n$ 
Table 1. Locomotion speed of A53T larvae at various concentrations of L-DOPA

\begin{tabular}{ccc}
$\begin{array}{c}\text { L-DOPA } \\
\text { conc. }(\boldsymbol{\mu M})\end{array}$ & $\begin{array}{c}\text { Crawling speed } \\
(\mathbf{m m} / \mathbf{m i n},+/-S E M)\end{array}$ & $\mathbf{n}$ \\
\hline 10 & $43.3+/-2.64$ & 6 \\
100 & $83.4+/-4.13$ & 18 \\
1,000 & $78.8+/-6.73$ & 6 \\
\hline
\end{tabular}

n, number of larvae tested.

(A53T) was expressed in DA neurons (TH-Gal4 x UAS-A53T). A53T larvae showed locomotion deficits in crawling speed, average angular velocity, and pause time (Fig. 1), demonstrating that A53T larva is a useful genetic animal model for Parkinson's disease (PD).

Since L-DOPA is a gold standard drug to ameliorate PD symptoms, we examined L-DOPA effects on locomotion defects in A53T larva. When L-DOPA $(100 \mu \mathrm{M})$ was administered in food, crawling speed of a-Syn (A53T) larvae was significantly increased (Fig. 1A, B). In addition, angular velocity and pause time were also rescued by L-DOPA (Fig. 1C, D). However, $10 \mu \mathrm{M}$ L-DOPA did not rescue the crawling speed in A53T larva (Table 1). We also observed that rescue effects of $1 \mathrm{mM} \mathrm{L-DOPA}$ were slightly reduced compared to $100 \mu \mathrm{M}$. Therefore, $100 \mu \mathrm{M}$ L-DOPA was used in these experiments to rescue locomotive symptoms as it has been determined to have the highest rescue effects in A53T Drosophila larvae. $100 \mu \mathrm{M}$ L-DOPA rescued speed deficits by $42.2 \%$, average angular velocity deficits by $75.3 \%$, and pause time deficits by $67 \%$ (Fig. 1). Our results demonstrated that treatment with $100 \mu \mathrm{M} \mathrm{L}$ DOPA rescued the locomotion defects of A53T larvae comparable to wild type levels.

\section{L-DOPA-induced dyskinesia in A53T larvae}

Because L-DOPA had experimentally shown to rescue Parkinsonian symptoms in Drosophila A53T larvae, it was hypothesized that excessive exposure to L-DOPA would induce dyskinetic behavior. Experiments were designed to induce dyskinetic behavior in Drosophila A53T larvae by overexposure to L-DOPA. We developed a method to quantify dyskinetic behavior in Drosophila larvae (Fig. 2A, B; also refer to Materials \& Methods section). In order to simulate the effects of prolonged exposure to L-DOPA, A53T larvae were treated with a high concentration (e.g, $10 \mathrm{mM}$ ) of L-DOPA for 92 hours and compared to wild type (WT), A53T and A53T larvae rescued with $100 \mu \mathrm{M}$ L-DOPA (Fig. 2C). In this study, we used $10 \mathrm{mM}$ L-DOPA to induce dyskinetic behavior, which is 100 times the rescuing amount. Exposing larvae to 10 $\mathrm{mM}$ L-DOPA showed body bending behavior (BBB; Fig. 2A), which mimics the axial dyskinesia, a symptom of dyskinetic be- havior in mammals $[16,22]$. Therefore, BBB was used as a parameter of LID to quantify dyskinetic behavior in this study. Larvae exposed to a high concentration $(10 \mathrm{mM})$ of L-DOPA increased BBB dramatically, but A53T larvae with $(\mathrm{A} 53 \mathrm{~T}+100 \mu \mathrm{M})$ or without (A53T Control) a low concentration of L-DOPA did not show changes in $\mathrm{BBB}$, demonstrating dyskinetic behaviors specifically in a genetic Drosophila model of PD exposed to a high concentration of L-DOPA.

\section{Inherent L-DOPA toxicity}

Because LID is not a symptom of PD, but rather a symptom of long-term exposure to L-DOPA, we hypothesized that there would be no significant difference in BBB of WT, A53T, or L-DOPA rescued larvae. In order to ensure that the change in body bending behavior is in fact due to L-DOPA-induced dyskinesia, and not due to inherent L-DOPA toxicity, various concentrations of L-DOPA were administered to WT larvae for 92 hours (Fig. 3A). The BBB of the WT larvae were then analyzed. Because WT larvae are not expressing $\boldsymbol{\alpha}$-synuclein, no PD-related dyskinetic behavior was expected when exposed to any levels of L-DOPA. Despite not expressing a-synuclein, however, WT larvae showed significantly higher levels of body bending behavior when exposed to high concentrations of L-DOPA (5 or $10 \mathrm{mM}$ ) although percent of $\mathrm{BBB}$ in WT larvae was lower compared to that of A53T+10 mM L-DOPA larvae. The results suggest that BBB observed in Fig. 2C is not in fact due to the isolation of LID, but involves, at least partly due to the inherent L-DOPA toxicity or drug-induced dyskinesia (see Discussion) possibly by an increase of neuronal DA levels.

\section{No inherent toxicity when WT larvae are exposed to $L$ - DOPA for 48 hours}

In order for the LID model to reliably isolate and quantify dyskinetic behavior, it was necessary to reduce the amount of time the flies were exposed to L-DOPA. This would minimize the toxic effects seen in WT flies exposed to high concentrations of LDOPA for 92 hours (Fig. 3B). All eggs were initially laid onto three control food plates. After incubating for the first and second instar stages ( 44 hours) without L-DOPA, larvae were then transferred to new food plates: WT, WT+100 $\mu \mathrm{M}$ L-DOPA, and WT+10 mM L-DOPA. The larvae on all three plates were then returned to the incubator for 48 more hours before testing. By limiting the exposure time, L-DOPA-dependent BBB seen in WT larvae with longterm exposure were eliminated (Fig. 3B). Therefore, this L-DOPA treatment protocol can be used to examine dyskinetic behavior in the A53T larval model of PD. 
A

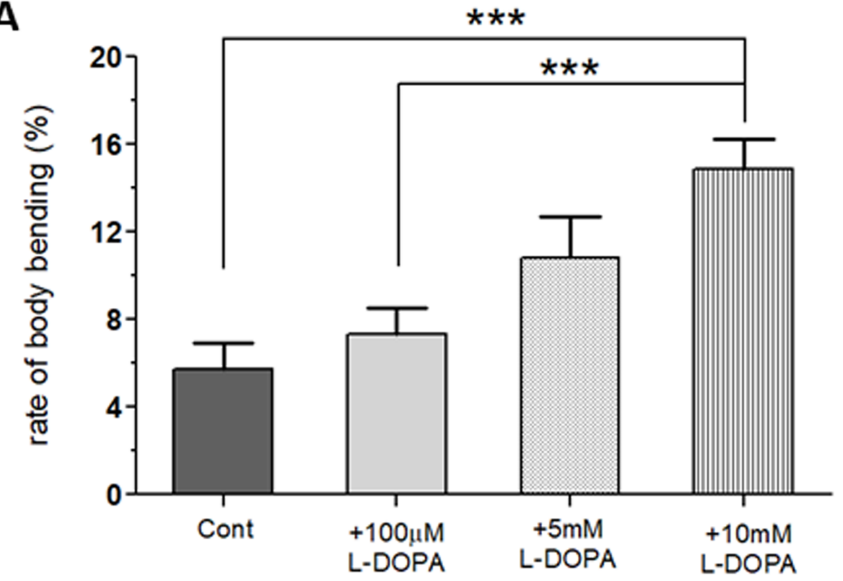

B

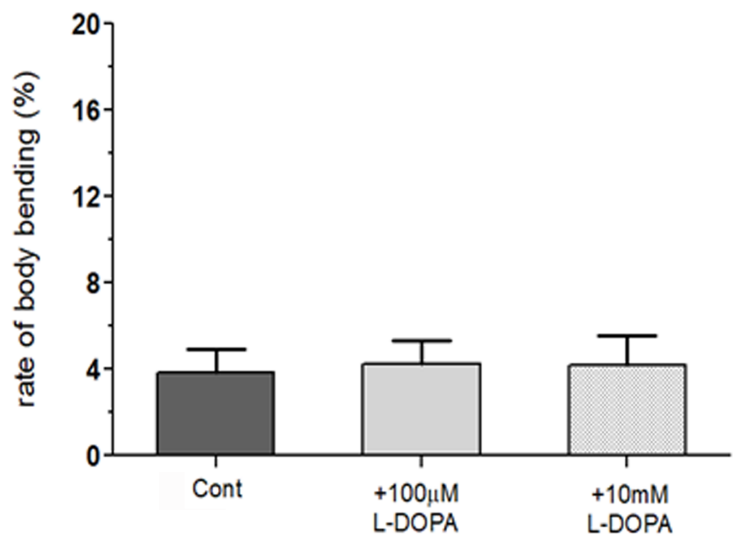

Fig. 3. Inherent toxicity of L-DOPA toxicity in wild type (WT) flies with longer exposure (92 hours), but not with shorter exposure (48 hours). (A) Wild type (WT) larvae treated with 5 or $10 \mathrm{mM} \mathrm{L-DOPA} \mathrm{showed} \mathrm{a} \mathrm{significant} \mathrm{increase} \mathrm{in} \mathrm{body} \mathrm{bending} \mathrm{behavior} \mathrm{(BBB)} \mathrm{over} \mathrm{WT} \mathrm{Control} \mathrm{when} \mathrm{L-DOPA}$ was treated from the first instar (for 92 hours). (B) WT larvae showed no changes in BBB when a high concentration (10 mM) of L-DOPA was exposed only during the third instar stage ( 48 hours). Student $\mathrm{t}$-test, ${ }^{* * *} \mathrm{p}<0.001$. For each group, 18 larvae from 3 separate experiments were examined.
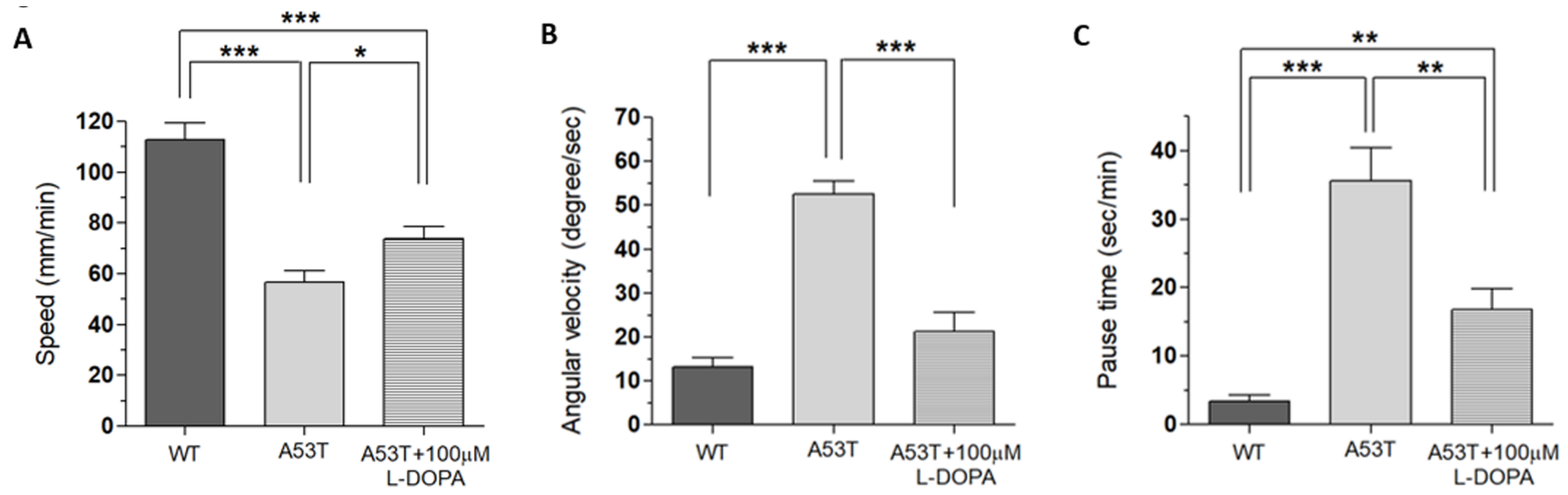

Fig. 4. Rescue of locomotion defects in A53T larvae with 48 hour exposure to $100 \mu \mathrm{M}$ L-DOPA. When treated with $100 \mu \mathrm{M}$ of L-DOPA for 48 hours, locomotion deficits in speed (A), angular velocity (B) and pause time (C) were significantly rescued. Locomotion deficits were not completely rescued by L-DOPA as there is still a significant difference between A53T+100 $\mu \mathrm{M} \mathrm{L}$-DOPA and WT Control. Student t-test, ${ }^{*} \mathrm{p}<0.05 ;{ }^{* *} \mathrm{p}<0.01 ;{ }^{* * *} \mathrm{p}<0.001$. For each group, 9 18 larvae from 3 separate experiments were examined.

\section{Rescue of locomotion defects in A53T larvae with 48 hour exposure to $100 \mu \mathrm{ML}$-DOPA}

Because WT larvae have shown no locomotion deficits (speed, angular velocity and pause time) when $100 \mu \mathrm{M}$ L-DOPA was applied for only 48 hours (data not shown), the next step was to test the rescue effects of $100 \mu \mathrm{M}$ L-DOPA when applied to A53T larvae for only 48 hours to make sure that L-DOPA could still rescue PD symptoms. The rescue effects of $100 \mu \mathrm{M}$ of L-DOPA were tested by applying for only 48 hours (Fig. 4). Even when applied for a shorter duration, L-DOPA still rescued the speed deficits of A53T larvae by $30.7 \%$, average angular velocity by $79.9 \%$, and pause time by $58.5 \%$. As far as body bending behavior was concerned, there was no difference between WT Control, A53T Control, and A53T larvae rescued with $100 \mu \mathrm{M}$ L-DOPA (Fig. 5A). The results prove that any behavior being measured by the BBB model is not a PD symptom, as it is not present in A53T larvae with or without $100 \mu \mathrm{M} \mathrm{L}$ DOPA.

\section{LID in A53T larvae with 48 hour exposure to $10 \mathrm{mM} \mathrm{L}$ - DOPA}

In this study, we examined whether the application of high doses of L-DOPA for 48 hours can induce dyskinetic behavior in Drosophila A53T larvae. The A53T larvae rescued with $100 \mu \mathrm{M}$ L-DOPA were compared to larvae treated with 5 and $10 \mathrm{mM} \mathrm{L-}$ 


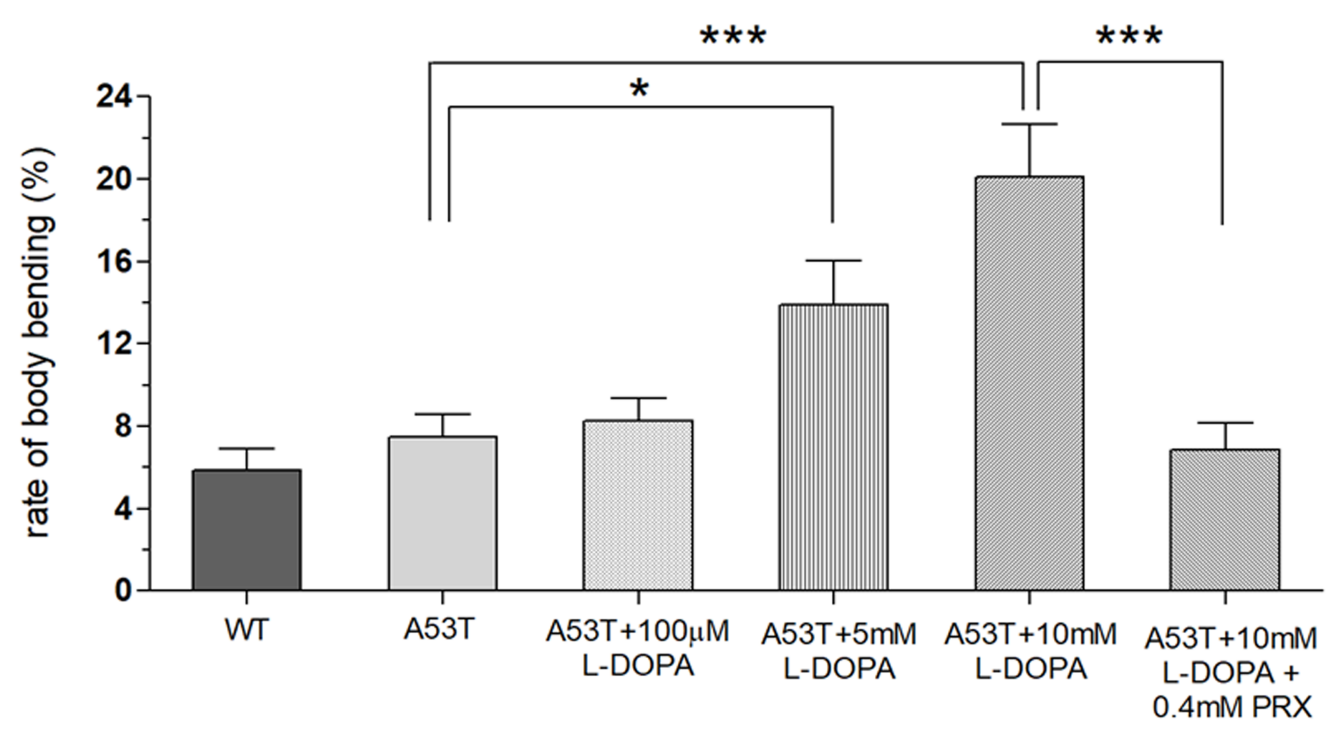

Fig. 5. Increased body bending behavior (BBB) in A53T larvae by shorter exposure (48 hours) to high concentrations of L-DOPA but rescued by a dopamine agonist pramipexole (PRX). BBB was increased in A53T larvae by high concentrations ( 5 or $10 \mathrm{mM}$ ) of L-DOPA exposure during the third instar (48 hours), but completely rescued when A53T larvae exposed to high concentrations of L-DOPA were treated with pramipexole (PRX, $0.4 \mathrm{mM}$ ). Student t-test, ${ }^{*} \mathrm{p}<0.05,{ }^{* * *} \mathrm{p}<0.001$. For each group, 18 larvae from 4 separate experiments were examined.

DOPA (Fig. 5). Exposure to 5mM L-DOPA increase BBB in A53T larvae by $172 \%$, and exposure to $10 \mathrm{mM}$ L-DOPA increased it in A53T larvae by $252 \%$, when compared to A53T larvae rescued by $100 \mu \mathrm{M}$ L-DOPA. Our data showed that high L-DOPA (5 or 10 $\mathrm{mM}$ ) significantly increases involuntary and hyperactive LID behaviors including body bending. In contrast, WT larvae showed no increase in body bending rate with $10 \mathrm{mM} \mathrm{L-DOPA}$ for 48 hours (Fig. 3B), confirming the successful development of a genetic LID model using Drosophila A53T larvae. This successful development of a Drosophila genetic LID model is very significant as LID is currently one of most difficult challenges in PD management.

\section{Rescue of LID with a dopamine agonist pramipexole}

With high L-DOPA proven to induce dyskinetic behavior in A53T larvae, the next step was to see if the symptoms of LID could be rescued. Pramipexole (PRX), a D2-like receptor agonist, was chosen, as it has been used in clinical settings on humans to treat LID $[23,24]$. Larvae were treated with $10 \mathrm{mM} \mathrm{L-DOPA}$ and 0.4 mM PRX for 48 hours and compared to A53T larvae exhibiting dyskinetic behavior for 48 hour-exposure to $10 \mathrm{mM}$ L-DOPA alone (Fig. 5). The administration of PRX fully rescued body bending behavior to the level similar to A53T without L-DOPA. PRX also rescued average angular velocity by $36.4 \%$ and pause time by $29.3 \%$. Interestingly the crawling speed was not rescued. The increase in angular velocity/pause time and decrease in speed by a high concentration of L-DOPA $(10 \mathrm{mM})$ can be explained by the loss of lateral movement due to $\mathrm{BBB}$. Our results suggest that PRX has potential to be a therapeutic agent for the treatment of $\mathrm{L}$ DOPA-induced dyskinesia in a genetic PD.

\section{DISCUSSION}

The leading treatment for Parkinson's disease symptoms is LDOPA, a precursor of dopamine, of which administration increases dopamine levels in the brain. But too much dopamine being present in the brain is expected to cause dysfunctional dopamine signaling. Prolonged exposure to L-DOPA can lead to L-DOPAinduced dyskinesia (LID) $[1,2,25]$. LID consists of involuntary, hyperactive behavior, and is a common occurrence among Parkinson's disease (PD) patients, yet there is currently no known cure and also no convenient way to screen potential therapeutic drugs. Complications of LID can be reduced by lowering the amount of L-DOPA that is taken, but unfortunately, this decrease in LDOPA can lead to a return of Parkinsonian symptoms. The current study is significant because it establishes a genetic LID model for the study of dyskinetic behavior. Depending on L-DOPA levels in brain and blood plasma, various patterns of LID have been characterized such as peak-dose LID, diphasic dyskinesia and off-period dystonia [26]. The most common pattern is peakdose LID which causes involuntary movements when L-DOPA level is high. On the basis of our method to administer L-DOPA, Drosophila larval LID model appears to represent peak-dose LID 
in mammals. Previously, there were few LID models which can be used to study underlying pathophysiological mechanisms, genetic/ molecular targets initiating the development of LID, and potential pharmacological therapies [27], largely due to lack of rich genetic resources. Recently, Gupta et al. [28] has been developed C. elegans LID model expressing human $\alpha$-synuclein, which shows increase body bending with L-DOPA. Overall, our Drosophila genetic LID model will provide an important experimental platform to examine molecular and cellular mechanisms underlying LID, to study the role of PD causing genes in the development of LID, and to identify potential targets to slow and/or reverse LID pathology. Therefore, this genetic LID model can greatly contribute our efforts to develop better therapeutic strategies and rapidly screen drugs alleviating PD symptoms without causing LID, providing significant translational impacts.

LID occur in 30 80\% of PD patients treated with L-DOPA [1,2, 54-56]. Two conditions are necessary for their appearance: 1) the loss of DA in the nigrostriatal pathway and 2) treatment with LDOPA $[55,56]$. In order to study LID, it should use a PD model showing the loss of DA neurons. Therefore, it is necessary to start with a PD model showing locomotion defects and degeneration of dopaminergic system, and then study its rescue with L-DOPA. LID can be developed using such PD model. The utilization of Drosophila in this study is particularly practical due to the established PD model available in fruit flies [11,29]. In addition, the relatively short lifespans of Drosophila make experimentation a quick process. Rather than waiting months to years for a neurodegenerative disease to present in mice, rats, or other animal models, experiments can be performed on larvae only four days after eggs are laid. In addition, the relatively low cost of maintenance and the large quantity in which they can be raised make the Drosophila model particularly accessible to labs across the world. Although adult fly is also widely used to study human neurodegenerative diseases, we used third instar larvae in this study because it is much easier to examine PD symptoms in terms of locomotive behavior. Also it takes less time to observe PD symptoms (4 days) and thus LID can be studied in this time frame.

Because of the longitudinal body shape of Drosophila larvae, axial dyskinesia is easily tracked. Axial dyskinesia has been effectively used to quantify LID in mice models [16], and therefore made sense as a parameter to be used in larva. The nature of the wells in the metal bar used to establish this model made tracking dyskinetic behavior along the longitudinal axis of the larvae particularly easy. The diameter of the wells is not much longer than the length of the larvae. This inhibits the larvae from any lateral crawling motion, but only allows two different degrees of motion. The first motion is rotation about the longitudinal axis of the larva, and the second motion is bending, or deviation, from the longitudinal axis of the larva (=involuntary movement in the fly larva). In future experiments, rotational movement could also be tracked [30]. In this study, axial dyskinesia was tracked, by comparing the distance between the head and the tail was compared to the length of the entire larva. Therefore, as the larva spends more time with its body contorted, bringing its head down to its tail, its percent body bending behavior goes up. This provides an easily identifiable and quantifiable method of measuring body bending behavior (BBB).

LID is an example of drug-induced dyskinesia (DID). Druginduced dyskinesia (e.g. Tardive dyskinesia or LID) is caused by the long-term use of a certain type of medications including antipsychotic drugs and levodopa [57]. It was interesting to observe that wild type larvae without PD symptoms showed dyskinetic behavior induced by high concentrations of L-DOPA (Fig. 3). It is possible to use wild type larva to study DID, but the current study focusses on LID which should be developed from a PD model with DA neurodegeneration in the brain. DID with wild type larva is certainly an interesting research topic to further explore.

Treatment of LID is still a major challenge. However, several drugs have been used to ameliorate LID including dopaminergic (e.g., DA-R agonist apomorphine) and non-dopaminergic drugs (e.g., NMDA receptor antagonist amantadine). Studies showed that DA receptor agonists can reduce $\operatorname{LID}[56,58,59]$. After successfully established fly larval model of LID, we tested a D2/3 agonist pramipexole to rescue LID in fly larvae as it has been shown pramipexole reduces dyskinesia in MPTP-treated primate when it was applied with L-DOPA $[23,60]$. Similarly, other known LID drugs (e.g., amantadine, safinamide) can be also studied for their therapeutic potentials on LID. Our model can be also used to screen new potential therapeutic drugs. Since we added pramipexole and L-DOPA concomitantly in the food, it certainly possible that pramipexole may cause neuroprotection against LID instead of rescuing LID. This possibility should be further examined in order to clearly show the rescue effects of pramipexole in the fly larval LID model.

It still remains to completely understand pathological mechanisms underlying LID $[31,32]$. Nonetheless, some potential mechanisms are related to alterations in DA signaling. Long-term exposure to L-DOPA alters neural circuits in basal ganglia [2, 4]. It has been suggested that uncontrollable release of dopamine (DA) by L-DOPA results in dyskinetic behaviors $[1,33]$. Altered activities of D1 and D2 receptors also cause abnormal movements by excessive cortical stimulation through L-DOPA-dependent disinhibition of thalamus [2]. Therefore, both PD and LID are caused by dysfunctional DA signaling although PD is due to reduced DA signaling while LID is by increased and uncoordinated DA activi- 
ties. Recently, it has been shown that hyperkinetic activity and altered D1 signaling underlie L-DOPA induced dyskinesia in C. elegans [28], further supporting the notion that uncontrollable DA signaling is a key pathological mechanism of LID. One important factor considering use of Drosophila model for LID is the similarity of DA signaling mechanisms in mammals and Drosophila. DA neurons in Drosophila receive both excitatory and inhibitory synaptic inputs and are spontaneously active. Drosophila DA autoreceptors also known to suppress the excitability [34-36]. Like mammals, excitatory dopamine D1-like and inhibitory D2-like receptors are expressed in Drosophila nervous system. Both Drosophila D1- (i.e. dDA1, DAMB) and D2-like (i.e. DD2R) receptors have been molecularly characterized [37-40]. Like in mammals, DA is known to play important roles in behavior such as locomotion, memory formation and drug addiction in Drosophila [41, 42]. Therefore, Drosophila LID model can be used to study pathological mechanisms underlying LID. One such signaling example is cAMP-PKA-CREB pathway, which has been intensively studied in Drosophila [43]. It is well known that cAMP signaling pathway is downstream of D1R signaling. Indeed, LID models using PD neurotoxins showed over-expression of Gaolf and adenylyl cyclase $V[44,45]$. It will be interesting to examine whether increased cAMP-PKA signaling is associated with our genetic LID model. Further, a chemogenetic approach (i.e., DREADD) [46, 47] can be used to manipulate intracellular cAMP levels.

In addition to L-DOPA, D2 agonists (e.g., pramipexole, bromocriptine, ropinirole) have been used clinically to treat PD symptoms [48]. We showed that activation of D2 receptors is critical to rescue dopaminergic (DA) degeneration by a PD toxin MPP+, and that D2 autoreceptors expressed in DA neurons are sufficient for this rescue [36]. For LID, few drugs are available to alleviate its symptoms at present, but clinical studies with PD patients showed that D2 agonists reduce the risk of dyskinesia incidence [24, 49, 50]. Among $D 2$ agonists, pramipexole is known to alleviate symptoms of LID in PD patients treated with L-DOPA [23]. D2 agonists are known to regulate the amount of DA release in the brain as they act mainly on presynaptic D2 receptors and thus inhibit DA release, counterbalancing increased levels of DA. Interestingly, we also observed rescue effects of a D2 agonist pramipexole (PRX) on LID in Drosophila a-Syn larvae (Fig. 5). PRX shows a strong binding affinity to D3 subtype, a type of D2-like receptors. So far, however, only one D2 receptor DD2R has been identified in Drosophila [40]. Therefore, it is assumed that Drosophila DD2R may be a common D2 receptor representing mammalian D2-like receptor subtypes - D2, D3 \& D4. Further, it has been shown that PRX improves motor symptoms in PD flies [51]. Therefore, role of D2like receptor family in LID can be further examined in Drosophila
a-Syn model. In addition, our larval LID model can be used to test the rescue effects of neuroprotective drugs, like nicotine and caffeine, on dyskinetic behavior [52]. Smoking and coffee drinking are known to protect against neurodegeneration, and thus prevent PD and Alzheimer's disease. For these reasons, Drosophila can be an effective and practical model to examine potential drugs for the rescue of LID.

Our study showed that low L-DOPA (100 $\mu \mathrm{M}$ in food) rescues PD motor symptoms in Drosophila larvae expressing mutant human $\alpha$-Syn ( $\alpha$-Syn larva) while higher L-DOPA $(10 \mathrm{mM})$ causes dyskinetic behaviors including involuntary and continuous body bending (similar to axial dyskinesia in rodents), demonstrating that Drosophila $\alpha$-Syn larvae can be an excellent genetic LID model. However, our current method is largely based on laborious manual analysis, limiting our quantification only to the bending behavior. It is desirable to automate and optimize the dyskinetic behavior analysis with high resolution images using a recently introduced method called 'MaggotTracker' - a single-animal tracking system for Drosophila larval locomotion [30]. This tracker system can quantify uncontrollable rotational behavior and peristalsis movement, which may be relevant to LID. MaggotTracker can also analyze free-moving larvae with high resolution images, instead of analyzing constrained larvae in a small well. In addition, it can analyze more behavioral phenotypes. LID in mammals is typically determined using sums of abnormal involuntary movement (AIM) scales: axial, limb and orolingual dyskinesias [53]. Further automation of LID analysis with more behavioral phenotypes (e.g, head bending, uncoordinated peristaltic movements) will enhance utilities of our Drosophila LID model to study its underlying pathological mechanisms and develop its therapeutic treatments.

In human, it takes several years to develop LID whereas it takes weeks to months for rodent models $[17,61]$. Although continuous administration of L-DOPA can induce dyskinesia in preclinical studies [62], most studies have reported an increase in the frequency of LID by extended period of L-DOPA use. Therefore, our fly model dose not exactly recapitulate what happened in mammals including human. However, we believe that our fly LID model is very useful to study molecular and cellular mechanisms underlying LID pathology as the primary cause of LID is initiated from the dysfunctional dopamine signaling. Drosophila has a conserved dopamine neural circuits which is known to play an important role in locomotion [42]. Further, LID in the fly larval model can be developed in 4 days and thus this model can be a very useful genetic platform to screen genes modifying LID pathology and also potential therapeutic LID drugs. There are limitations in the fly LID model but it has other advantages over mammalian LID models. 


\section{ACKNOWLEDGEMENTS}

This work was partially supported by the International Collaboration Grant from Korea Institute of Science \& Technology (Brain Science Institute), Seoul, Korea. We thank Jason Bury at University of Toledo for helping us to develop a software for locomotion analysis. JAB was a recipient of Ohio University Provost's Undergraduate Research Fund.

\section{REFERENCES}

1. Espay AJ, Morgante F, Merola A, Fasano A, Marsili L, Fox SH, Bezard E, Picconi B, Calabresi P, Lang AE (2018) Levodopainduced dyskinesia in Parkinson disease: current and evolving concepts. Ann Neurol 84:797-811.

2. Lane EL (2019) L-DOPA for Parkinson's disease-a bittersweet pill. Eur J Neurosci 49:384-398.

3. Morin N, Jourdain VA, Di Paolo T (2014) Modeling dyskinesia in animal models of Parkinson disease. Exp Neurol 256:105-116.

4. Bastide MF, Meissner WG, Picconi B, Fasano S, Fernagut PO, Feyder M, Francardo V, Alcacer C, Ding Y, Brambilla R, Fisone G, Jon Stoessl A, Bourdenx M, Engeln M, Navailles S, De Deurwaerdère P, Ko WK, Simola N, Morelli M, Groc L, Rodriguez MC, Gurevich EV, Quik M, Morari M, Mellone M, Gardoni F, Tronci E, Guehl D, Tison F, Crossman AR, Kang UJ, Steece-Collier K, Fox S, Carta M, Angela Cenci M, Bézard E (2015) Pathophysiology of L-dopa-induced motor and non-motor complications in Parkinson's disease. Prog Neurobiol 132:96-168.

5. Dawson TM, Ko HS, Dawson VL (2010) Genetic animal models of Parkinson's disease. Neuron 66:646-661.

6. Poewe W, Seppi K, Tanner CM, Halliday GM, Brundin P, Volkmann J, Schrag AE, Lang AE (2017) Parkinson disease. Nat Rev Dis Primers 3:17013.

7. Fleming SM, Fernagut PO, Chesselet MF (2005) Genetic mouse models of Parkinsonism: strengths and limitations. NeuroRx 2:495-503.

8. Beal MF (2010) Parkinson's disease: a model dilemma. Nature 466:S8-S10.

9. Adams MD, Celniker SE, Holt RA, Evans CA, Gocayne JD, Amanatides PG, Scherer SE, Li PW, Hoskins RA, Galle RF, George RA, Lewis SE, Richards S, Ashburner M, Henderson SN, Sutton GG, Wortman JR, Yandell MD, Zhang Q, Chen LX, Brandon RC, Rogers YH, Blazej RG, Champe M, Pfeiffer BD, Wan KH, Doyle C, Baxter EG, Helt G, Nelson CR, Gabor GL, Abril JF, Agbayani A, An HJ, Andrews-Pfannkoch C, Baldwin
D, Ballew RM, Basu A, Baxendale J, Bayraktaroglu L, Beasley EM, Beeson KY, Benos PV, Berman BP, Bhandari D, Bolshakov S, Borkova D, Botchan MR, Bouck J, Brokstein P, Brottier P, Burtis KC, Busam DA, Butler H, Cadieu E, Center A, Chandra I, Cherry JM, Cawley S, Dahlke C, Davenport LB, Davies P, de Pablos B, Delcher A, Deng Z, Mays AD, Dew I, Dietz SM, Dodson K, Doup LE, Downes M, Dugan-Rocha S, Dunkov BC, Dunn P, Durbin KJ, Evangelista CC, Ferraz C, Ferriera S, Fleischmann W, Fosler C, Gabrielian AE, Garg NS, Gelbart WM, Glasser K, Glodek A, Gong F, Gorrell JH, Gu Z, Guan P, Harris M, Harris NL, Harvey D, Heiman TJ, Hernandez JR, Houck J, Hostin D, Houston KA, Howland TJ, Wei MH, Ibegwam C, Jalali M, Kalush F, Karpen GH, Ke Z, Kennison JA, Ketchum KA, Kimmel BE, Kodira CD, Kraft C, Kravitz S, Kulp D, Lai Z, Lasko P, Lei Y, Levitsky AA, Li J, Li Z, Liang Y, Lin X, Liu X, Mattei B, McIntosh TC, McLeod MP, McPherson D, Merkulov G, Milshina NV, Mobarry C, Morris J, Moshrefi A, Mount SM, Moy M, Murphy B, Murphy L, Muzny DM, Nelson DL, Nelson DR, Nelson KA, Nixon K, Nusskern DR, Pacleb JM, Palazzolo M, Pittman GS, Pan S, Pollard J, Puri V, Reese MG, Reinert K, Remington K, Saunders RD, Scheeler F, Shen H, Shue BC, Sidén-Kiamos I, Simpson M, Skupski MP, Smith T, Spier E, Spradling AC, Stapleton M, Strong R, Sun E, Svirskas R, Tector C, Turner R, Venter E, Wang AH, Wang X, Wang ZY, Wassarman DA, Weinstock GM, Weissenbach J, Williams SM, WoodageT, Worley KC, Wu D, Yang S, Yao QA, Ye J, Yeh RF, Zaveri JS, Zhan M, Zhang G, Zhao Q, Zheng L, Zheng XH, Zhong FN, Zhong W, Zhou X, Zhu S, Zhu X, Smith HO, Gibbs RA, Myers EW, Rubin GM, Venter JC (2000) The genome sequence of Drosophila melanogaster. Science 287:2185-2195.

10. Chien S, Reiter LT, Bier E, Gribskov M (2002) Homophila: human disease gene cognates in Drosophila. Nucleic Acids Res 30:149-151.

11. Feany MB, Bender WW (2000) A Drosophila model of Parkinson's disease. Nature 404:394-398.

12. Auluck PK, Chan HY, Trojanowski JQ, Lee VM, Bonini NM (2002) Chaperone suppression of alpha-synuclein toxicity in a Drosophila model for Parkinson's disease. Science 295:865868.

13. Trinh K, Moore K, Wes PD, Muchowski PJ, Dey J, Andrews L, Pallanck LJ (2008) Induction of the phase II detoxification pathway suppresses neuron loss in Drosophila models of Parkinson's disease. J Neurosci 28:465-472.

14. Park SS, Lee D (2006) Selective loss of dopaminergic neurons and formation of Lewy body-like aggregations in alphasynuclein transgenic fly neuronal cultures. Eur J Neurosci 


\section{3:2908-2914.}

15. Varga SJ, Qi C, Podolsky E, Lee D (2014) A new Drosophila model to study the interaction between genetic and environmental factors in Parkinson's disease. Brain Res 1583:277286.

16. Urs NM, Bido S, Peterson SM, Daigle TL, Bass CE, Gainetdinov RR, Bezard E, Caron MG (2015) Targeting $\beta$-arrestin2 in the treatment of L-DOPA-induced dyskinesia in Parkinson's disease. Proc Natl Acad Sci U S A 112:E2517-E2526.

17. Alberico SL, Kim YC, Lence T, Narayanan NS (2017) Axial levodopa-induced dyskinesias and neuronal activity in the dorsal striatum. Neuroscience 343:240-249.

18. Friggi-Grelin F, Coulom H, Meller M, Gomez D, Hirsh J, Birman S (2003) Targeted gene expression in Drosophila dopaminergic cells using regulatory sequences from tyrosine hydroxylase. J Neurobiol 54:618-627.

19. Davis MY, Trinh K, Thomas RE, Yu S, Germanos AA, Whitley BN, Sardi SP, Montine TJ, Pallanck LJ (2016) Glucocerebrosidase deficiency in Drosophila results in a-synucleinindependent protein aggregation and neurodegeneration. PLoS Genet 12:e1005944.

20. Chen AY, Wilburn P, Hao X, Tully T (2014) Walking deficits and centrophobism in an $a$-synuclein fly model of Parkinson's disease. Genes Brain Behav 13:812-820.

21. Brand AH, Perrimon N (1993) Targeted gene expression as a means of altering cell fates and generating dominant phenotypes. Development 118:401-415.

22. Solís O, Garcia-Montes JR, González-Granillo A, Xu M, Moratalla R (2017) Dopamine D3 receptor modulates 1-DOPAinduced dyskinesia by targeting D1 receptor-mediated striatal signaling. Cereb Cortex 27:435-446.

23. Piedad JC, Cavanna AE (2012) Dyskinesias and treatment with pramipexole in patients with Parkinson's disease. Parkinsons Dis 2012:473769.

24. Utsumi H, Okuma Y, Kano O, Suzuki Y, Iijima M, Tomimitsu H, Hashida H, Kubo S, Suzuki M, Nanri K, Matsumura M, Murakami H, Hattori N; Tokyo Parkinson's Disease Study Group (2013) Evaluation of the efficacy of pramipexole for treating levodopa-induced dyskinesia in patients with Parkinson's disease. Intern Med 52:325-332.

25. Jankovic J, Aguilar LG (2008) Current approaches to the treatment of Parkinson's disease. Neuropsychiatr Dis Treat 4:743757.

26. Cenci MA, Crossman AR (2018) Animal models of 1-dopainduced dyskinesia in Parkinson's disease. Mov Disord 33:889-899.

27. Iderberg H, Francardo V, Pioli EY (2012) Animal models of L-
DOPA-induced dyskinesia: an update on the current options. Neuroscience 211:13-27.

28. Gupta DK, Hang X, Liu R, Hasan A, Feng Z (2016) Levodopainduced motor and dopamine receptor changes in caenorhabditis elegans overexpressing human alpha-synuclein. Neurodegener Dis 16:179-183.

29. Xiong Y, Yu J (2018) Modeling Parkinson's disease in Drosophila: what have we learned for dominant traits? Front Neurol 9:228.

30. Aleman-Meza B, Jung SK, Zhong W (2015) An automated system for quantitative analysis of Drosophila larval locomotion. BMC Dev Biol 15:11.

31. Jenner P (2008) Molecular mechanisms of L-DOPA-induced dyskinesia. Nat Rev Neurosci 9:665-677.

32. Fisone G, Bezard E (2011) Molecular mechanisms of 1-DOPA-induced dyskinesia. Int Rev Neurobiol 98:95-122.

33. Heumann R, Moratalla R, Herrero MT, Chakrabarty K, Drucker-Colín R, Garcia-Montes JR, Simola N, Morelli M (2014) Dyskinesia in Parkinson's disease: mechanisms and current non-pharmacological interventions. J Neurochem 130:472-489.

34. Tepper JM, Lee CR (2007) GABAergic control of substantia nigra dopaminergic neurons. Prog Brain Res 160:189-208.

35. Vickrey TL, Venton BJ (2011) Drosophila dopamine2-like receptors function as autoreceptors. ACS Chem Neurosci 2:723-729.

36. Wiemerslage L, Schultz BJ, Ganguly A, Lee D (2013) Selective degeneration of dopaminergic neurons by $\mathrm{MPP}(+)$ and its rescue by D2 autoreceptors in Drosophila primary culture. J Neurochem 126:529-540.

37. Gotzes F, Balfanz S, Baumann A (1994) Primary structure and functional characterization of a Drosophila dopamine receptor with high homology to human D1/5 receptors. Recept Channels 2:131-141.

38. Feng G, Hannan F, Reale V, Hon YY, Kousky CT, Evans PD, Hall LM (1996) Cloning and functional characterization of a novel dopamine receptor from Drosophila melanogaster. J Neurosci 16:3925-3933.

39. Han KA, Millar NS, Grotewiel MS, Davis RL (1996) DAMB, a novel dopamine receptor expressed specifically in Drosophila mushroom bodies. Neuron 16:1127-1135.

40. Hearn MG, Ren Y, McBride EW, Reveillaud I, Beinborn M, Kopin AS (2002) A Drosophila dopamine 2-like receptor: molecular characterization and identification of multiple alternatively spliced variants. Proc Natl Acad Sci U S A 99:14554-14559.

41. Riemensperger T, Völler T, Stock P, Buchner E, Fiala A (2005) 
Punishment prediction by dopaminergic neurons in Drosophila. Curr Biol 15:1953-1960.

42. Rothenfluh A, Heberlein U (2002) Drugs, flies, and videotape: the effects of ethanol and cocaine on Drosophila locomotion. Curr Opin Neurobiol 12:639-645.

43. Lee D (2015) Global and local missions of cAMP signaling in neural plasticity, learning, and memory. Front Pharmacol 6:161.

44. Aubert I, Guigoni C, Håkansson K, Li Q, Dovero S, Barthe N, Bioulac BH, Gross CE, Fisone G, Bloch B, Bezard E (2005) Increased D1 dopamine receptor signaling in levodopainduced dyskinesia. Ann Neurol 57:17-26.

45. Rangel-Barajas C, Silva I, Lopéz-Santiago LM, Aceves J, Erlij D, Florán B (2011) L-DOPA-induced dyskinesia in hemiparkinsonian rats is associated with up-regulation of adenylyl cyclase type V/VI and increased GABA release in the substantia nigra reticulata. Neurobiol Dis 41:51-61.

46. Becnel J, Johnson O, Majeed ZR, Tran V, Yu B, Roth BL, Cooper RL, Kerut EK, Nichols CD (2013) DREADDs in Drosophila: a pharmacogenetic approach for controlling behavior, neuronal signaling, and physiology in the fly. Cell Rep 4:1049-1059.

47. Sternson SM, Roth BL (2014) Chemogenetic tools to interrogate brain functions. Annu Rev Neurosci 37:387-407.

48. Perez-Lloret S, Rascol O (2010) Dopamine receptor agonists for the treatment of early or advanced Parkinson's disease. CNS Drugs 24:941-968.

49. Rascol O, Brooks DJ, Korczyn AD, De Deyn PP, Clarke CE, Lang AE (2000) A five-year study of the incidence of dyskinesia in patients with early Parkinson's disease who were treated with ropinirole or levodopa. N Engl J Med 342:1484-1491.

50. Rascol O, Brooks DJ, Korczyn AD, De Deyn PP, Clarke CE, Lang AE, Abdalla M; 056 Study Group (2006) Development of dyskinesias in a 5-year trial of ropinirole and L-dopa. Mov Disord 21:1844-1850.

51. Siddique YH, Naz F, Khan W, Jyoti S, Singh BR, Naqvi AH (2018) Effect of pramipexole alginate nanodispersion (PAND) on the transgenic Drosophila expressing human alpha synuclein in the brain. J Appl Biomed 16:111-119.

52. Ross GW, Petrovitch H (2001) Current evidence for neuroprotective effects of nicotine and caffeine against Parkinson's disease. Drugs Aging 18:797-806.

53. Lindgren HS, Lane EL (2011) Rodent models of L-DOPA-induced dyskinesia. In: Animal models of movement disorders (Lane EL, Dunnett SB, eds), pp 337-351. Humana Press, New York.

54. Nutt JG, Woodward WR, Carter JH, Gancher ST (1992) Effect of long-term therapy on the pharmacodynamics of levodopa. Relation to on-off phenomenon. Arch Neurol 49:1123-1130.

55. Pandey S, Srivanitchapoom P (2017) Levodopa-induced dyskinesia: clinical features, pathophysiology, and medical management. Ann Indian Acad Neurol 20:190-198.

56. Sharma S, Singh S, Sharma V, Singh VP, Deshmukh R (2015) Neurobiology of 1-DOPA induced dyskinesia and the novel therapeutic strategies. Biomed Pharmacother 70:283-293.

57. Damier P (2009) Drug-induced dyskinesias. Curr Opin Neurol 22:394-399.

58. Deleu D, Hanssens Y, Northway MG (2004) Subcutaneous apomorphine: an evidence-based review of its use in Parkinson's disease. Drugs Aging 21:687-709.

59. Thanvi B, Lo N, Robinson T (2007) Levodopa-induced dyskinesia in Parkinson's disease: clinical features, pathogenesis, prevention and treatment. Postgrad Med J 83:384-388.

60. Tayarani-Binazir KA, Jackson MJ, Rose S, Olanow CW, Jenner P (2010) Pramipexole combined with levodopa improves motor function but reduces dyskinesia in MPTP-treated common marmosets. Mov Disord 25:377-384.

61. Tomas D, Stanic D, Chua HK, White K, Boon WC, Horne M (2016) Restoration of the dopamine transporter through cell therapy improves dyskinesia in a rat model of Parkinson's disease. PLoS One 11:e0153424.

62. Koller WC, Hutton JT, Tolosa E, Capilldeo R (1999) Immediate-release and controlled-release carbidopa/levodopa in PD: a 5-year randomized multicenter study. Carbidopa/Levodopa Study Group. Neurology 53:1012-1019. 